and nuclear physics. There need be no lowering of standards to achieve this end; there is a great deal in classical physies which makes a high call upon the mental capacity of the student, and which is, moreover, of considerable importance to the experimentalist, even if he is concerned with some of the newer branches of physics.

It is intended that instruction shall, whenever possible, be illustrated by practical usage. For example, optics will include practical photometry and the theory of stops leading to the design of optical systems; in magnetism, attention will be directed to the potentialities of materials with high coercivities and high initial permeabilities; and in heat, methods of measurement of high temperatures will be dealt with. Where possible, specialist lectures on such topics will be arranged.

The supporting subjects have been chosen with the same end in view. The mathematics will be mainly that which has applications in physics; some chemistry and metallurgy will be included for their importance in the theory of interatomic forces; and electrical engineering will supply the knowledge necessary for the handling of electrical instruments and supplies. Electronics will be dealt with particularly thoroughly, as it forms an important part of the equipment of the practical physicist.

The more promising students will be encouraged, after graduation, to take part in the research work of the Physics Department of the College of Tech. nology. At present, this is concerned with the study of crystalline matter by X-ray diffraction, a subject that is particularly apt since, in addition to its importance in fundamental physics, it has many applications in industry ; the work of the Department in this field is well known, both in Great Britain and abroad. Lack of space does not allow a wider choice of research subjects, but, as the Department expands, it is hoped to widen the research interests to include other subjects of practical importance.

It will be seen, therefore, that the intention is to provide a broadly based course that will fit students for a wide variety of applications of physics. If such students have certain gaps in their knowledge of the most recent advances in physics, it is hoped the breadth of the foundations laid will enable them to cope successfully with the difficulties they may encounter in their subsequent careers.

\section{METABOLISM OF GLUTATHIONE}

\section{By Prof. FRANCIS BINKLEY}

College of Medicine, University of Utah, Salt Lake City

$\mathrm{T}$ HE report of Hanes, Hird and Isherwood in Nature $^{1}$ of certain transpeptidation reactions with glutathione has prompted us to describe briefly certain of our findings and concepts of a similar nature. Several years ago we initiated an investigation of the metabolism of glutathione in the belief that this metabolism was concerned with the synthesis of protein. It was expected that the synthetic reactions would be found to be associated with reactions leading to the hydrolysis of glutathione, and, therefore, an investigation of the enzymes responsible for the hydrolysis of glutathione was undertaken. As we have reported ${ }^{2}$, it was found that two consecutive reactions were concerned with the hydrolysis by renal tissue; these reactions were ascribed to two separate enzymes, glutathionase and cysteinylglycinase, respectively. Cysteinylglycinase was found in all tissues other than nervous tissue, but glutathionase was found in reasonable quantities only in the kidney and intestine. This limited distribution of glutathionase, together with other considerations, led us to abandon the concept that the hydrolysis of glutathione was a specific mechanism concerned with protein synthesis, and caused us to consider the possibility that the hydrolysis was intimately concerned with absorptive processes and, in particular, with the function of the intestine and the kidney. It is our concept that although the reactions of glutathione may be a fragment of the mechanism of protein synthesis, it is specifically adapted to absorptive processes. Thus, we have pictured the renal tubule as composed essentially of a column of self-regenerating ionexchange resin; this 'resin' would be composed of the enzymes concerned with the hydrolysis of glutathione and glutamine ${ }^{3}$. The involvement of glutamine in the mechanism was prompted by our finding that the hydrolysis of glutathione was dependent upon the presence of glutamine. The reactions we have proposed to explain the relationship of glutamine and regeneration of the ion-exchange system are as follows :

(1) Enzyme-COONa + glutamine $\rightarrow$

Enzyme-CONH ${ }_{2}+\mathrm{Na}$ glutamate.

(2) Enzyme-CONH$H_{2}+$ glutathione $\rightarrow$ Enzyme-

$\mathrm{COOH}+$ glutamine + cysteinylglycine.

The 'ion-exchange resin' is thought to be the 'enzyme-COOH' and the regeneration process would be the shift of the amide grouping; the resynthesis of glutathione may proceed through reactions involving phosphate. Thus, it is seen that our concept of an amide transfer is very similar to that proposed by Hanes, Hird and Isherwood for 'transpeptidation' reactions. The role of glutamine in the formation of urinary ammonia ${ }^{5}$ could be explained by a simple extension of these concepts. The involvement of an 'ammonia-glutamic acidglutamine-glutathione' system in absorptive processes and in the maintenance of the ionic environ. ment of the animal has received indirect support from other laboratories. For example, Terner, Eggleston and $\mathrm{Krebs}^{6}$ have found that glutamate appears to be concerned with the maintenance of levels of potassium by retina and by nervous tissue, and Grunert and Phillips ${ }^{7}$ have reported that the levels of sodium in the bloods of alloxan-diabetic rats receiving diets low in salt are correlated with the levels of glutathione in the blood. Recent studies in our laboratory have shown that the administration of glutamine and glutathione to dogs in the course of a saline diuresis was followed by a marked decrease of excretion of sodium chloride. Furthermore, it has been found that the concentration of glutathione and the products of its hydrolysis in tissues of rats was changed markedly by the administration of insulin and deoxycorticosterone acetate; the effects of insulin appeared to be primarily upon the liver and muscle, whereas the effects of deoxycorticosterone acetate were primarily upon kidney. Such results are of interest in view of current concepts that these materials alter the ionic environment of tissues.

In view of our concept that this system is concerned with renal function, it is of interest that phenol red, $p$-aminohippurate, diodrast and penicillin were found to be competitive inhibitors of the hydro. lysis of glutathione. Since all these materials are 
known to be secreted by the kidney, it would appear possible that these inhibitions are related to the process of secretion. For this reason and because a variety of phthaleins were found to be more potent inhibitors than penicillin, we do not believe that the inhibition by penicillin can be considered as an explanation of the antibiotic properties of penicillin. The order of activity of the phthaleins as inhibitors of the hydrolysis was roughly the same as their order of activity as laxatives, thus re-emphasizing the possibility that the action of certain laxatives and secretion by the kidney may have a similar basis.

Further studies of the enzymes responsible for the hydrolysis have centred upon an elucidation of the nature of the cysteinylglycinase. As we have reported $^{8}$, there is reason to suspect that this enzyme is not protein in nature and, indeed, may be a pentose nucleic acid. The evidence for the non-protein nature of the enzyme is as follows: (1) activity was not reduced by long periods of incubation with relatively massive amounts of cyanide-papain, pepsin, trypsin or chymotrypsin; (2) substantially all the protein was removed by exhaustive treatment with chloroform and octanol and by fractionation with ethanol and salts without loss of activity ; (3) protein could not be detected in amounts of material as great as $10 \mathrm{mgm}$., whereas $0.0001 \mathrm{mgm}$. was sufficient for easily demonstrated activity (50 per cent hydrolysis in 15 min.); (4) injection of the material into rabbits did not induce the formation of antibodies which could be precipitated in the presence of the enzyme, could fix complement in the presence of the enzyme or inhibit the activity of the enzyme; and (5) the spectrum of the material in the ultra-violet and the content of nitrogen, pentose and phosphate were identical with that ordinarily accepted for pentose nucleic acids. It is to be emphasized that throughout the isolation and throughout such procedures as chromatography on ion-exchange resins, the activity was found to bear a constant relationship to the content of pentose and the absorption at $260 \mu$. The activity was extremely labile at values of $p \mathbf{H}$ less than $5 \cdot 5$, but solutions could be heated at $70^{\circ}$ for $10 \mathrm{~min}$. when the $p \mathrm{H}$ was about 9 ; at values of $p \mathbf{H}$ greater than $10 \cdot 5$, the activity was rapidly destroyed. The original material was attacked very slowly by ribonuclease, whereas the acid- or alkalineinactivated material was attacked rapidly.

Glutathionase appears to be a lipoprotein which may be extracted with $1 M$ sodium chloride or with alkaline buffers from saline-washed homogenates of kidney tissue. The material was precipitated from $1 M$ sodium chloride when the extract was diluted with water. The preparation was freed from deoxyribonucleic acid by washing with water and by flotation from $0.5 M$ sodium chloride. This material was inactive in the absence of glutamine and was activated considerably by cysteinylglycinase.

An application to tissues ${ }^{9}$ of our methods for the determination of glutathione and the products of its hydrolysis led to the discovery of an acid-labile derivative of cysteine which appeared to be $\gamma$-glutamylcysteine. Since our scheme for the hydrolysis of glutathione by enzymes of tissues had failed to predict such a product of hydrolysis, we have undertaken a search for the enzyme or enzymes responsible for the formation of this product. The only enzyme found that catalysed such a reaction was crystalline pancreatic carboxypeptidase. 'It may be possible that the activity of this enzyme (or of similar enzymes in other tissues) will explain the presence of $\gamma$-glutam- yleysteine in tissues; but our search is now directed toward the detection of systems concerned with a transfer of glycine from glutathione with the forma. tion of $\gamma$-glutamyleysteine. It is to be mentioned that this fraction has been found to be increased markedly in the bloods of diabetic patients ${ }^{9}$ and in the bloods of normals following the administration of adreno. corticotrophic hormone.

Thus, it is apparent that the metabolism of glutathione has wide ramifications in a number of physiological processes ; the elucidation of this metabolism has presented many fascinating and difficult problems of concept as well as of technique. It is a source of considerable satisfaction that the studies of the metabolism of glutathione have passed beyond the somewhat sterile concepts of the role of glutathione as a sulphhydryl or redox buffer and as a model for the study of formation of peptide bonds; it is hoped that the studies of the mechanisms of utilization of glutathione concerned with the hydrolysis will be somewhat more productive. ${ }^{1}$ Hanes, C. S., Hird, F. J. K., and Isherwood, F. A., Nature, 168, 288

2 Binkley, F., and Nakamura, K., J. Biol. Chem., 178, 411 (1948).

$s$ Binkley, F., in First Conference on Renal Function, New York (1949). ${ }^{4}$ Binkley, F., and Olson, C. K., J. Biol. Chem., 188, 451 (1951).

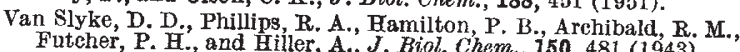
- Terner, C., Eggleston, L. V., and Krebs, H. A., Biochem (1943). $(1950)$.

${ }^{7}$ Grunert, R. R., and Phillips, P. H., J. Biol. Chem., 181, 821 (1949). Binkley, F., and Olson, C. K. Abstracts, 118th Meeting, American
Chemical Society, Chicago (1950).

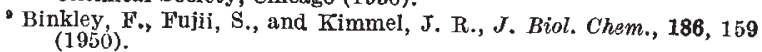

\section{DISTRIBUTION OF RADIATION ACROSS THE SOLAR DISK AT A FREQUENCY OF $81.5 \mathrm{MC}$. $/ \mathrm{S}$.}

\author{
By K. E. MACHIN
}

Cavendish Laboratory, Cambridge

TN a previous communication from this Laboratory, 1 Stanier ${ }^{1}$ has described how he used an interference method to measure the distribution of 'radio brightness' across the solar disk at a frequency of $500 \mathrm{Mc} . / \mathrm{s}$. In the first part of this note it is explained how the method has been used for measuring this distribution at a frequency of $81.5 \mathrm{Mc} . / \mathrm{s}$.; in the second part, the results obtained at both frequencies are considered in relation to existing theories of radio emission from the solar corona.

The principle of Stanier's method is as follows : two aerials, many wave-lengths apart, are connected to the same receiver, so that a recorder, operated by the output of the receiver, draws out a periodic 'interference pattern' as the reception pattern of the aerials is swept across the sun by the rotation of the earth. The amplitude of the pattern will vary with the spacing of the aerials, and from the nature of this variation it is possible to deduce the distribution of brightness across the solar disk.

In the present experiment, at a frequency of $81.5 \mathrm{Mc} / \mathrm{s}$. , four fixed and two movable aerials were used, so as to allow records to be obtained with twelve different aerial spacings. The gains and reception patterns of the aerials were previously measured by recording the radiation from the intense radio stars 05.01 and $12.01^{2}$ (in the constellations of Taurus and Virgo respectively). An 